\title{
Electrogastrographic responses to the stress of a mirror drawing test in outpatients consulting a psychosomatic clinic
}

\author{
Shinji HOMMA ${ }^{1}$, Ichiro MASHIMA², \\ Yoshiyuki MURAMATSU ${ }^{3}$ and Fumitake GEJYO ${ }^{2}$ \\ ${ }^{1}$ Division of Physiology II, Faculty of Medicine; \\ ${ }^{2}$ Division of Internal Medicine II, Faculty of Medicine; \\ ${ }^{3}$ Division of Nursing School of Health Sciences, Niigata University Graduate School of \\ Medical and Dental Sciences, Japan
}

Received June 8, 2009; Accepted August 1, 2009

\begin{abstract}
The respiratory rate, $\mathrm{LF}, \mathrm{HF}$, and the $\mathrm{LF} / \mathrm{HF}$ ratio from heart rate variability (HRV) were compared between outpatients $(n=25)$ and controls $(n=58)$ during rest and while performing a mirror drawing test (MDT). Anxiety and depression scores were obtained before the test. In addition, the power content of the electrogastrograms (EGGs) recorded during MDT were compared to those recorded during rest (c-MDT/r). The anxiety scores, depression scores, resting heart rate, resting respiratory rate and the 3 cpm resting frequency of the epigastric EGG were significantly higher in outpatients than in controls. Both the heart rate and the $3 \mathrm{cpm}$ frequency in the infraumbilical EGG during the stress of the MDT were significantly higher in outpatients than in controls. Instability factors (IF, standard deviation/mean frequency) of the $3 \mathrm{cpm}$ frequency in the epigastric EGG were higher in controls than in outpatients, though the spectral frequencies were similar and insignificantly different between the two groups. Interestingly, the slope of the significant linear correlation was positive in controls but was negative in outpatients between both the resting $\mathrm{LF} / \mathrm{HF}$ and the power content ratio of the $3 \mathrm{cpm}$ frequency in the infraumbilical EGG, c-MDT/r, which corresponds to the colonic responses to MDT stress.
\end{abstract}

Key words: electrogastrography, electrogastrogram (EGG), stress, mirror drawing test (MDT)

\section{Introduction}

The correlations between the anxiety scores, depression scores, HRV, respiratory rate and the EGG indices of spectral frequencies, power ratio, IF during rest and the acute stress during the MDT have been investigated in normal controls (Homma, 2005; Homma, 2006). In this

Correspondence to: Dr. Shinji Homma, MD, Ph.D., Niigata Shinai Hospital, Kamishinei-cho, Nishi-ku,

Niigata City, Niigata 950-2076, Japan

Phone: +81-25-269-4101 Fax: +81-25-260-4388 e-mail: sjnhomma@shinai.jp 
study we have compared those indices between control subjects and patients suffering from psychosomatic or psychiatric diseases. A comparison was made between normal controls and outpatients consulting the Clinic of Psychosomatic Medicine of Niigata University Medical and Dental Hospital. Anxiety scores, depression scores, resting heart and respiratory rate, and the $3 \mathrm{cpm}$ of the EGG spectral frequency were significantly higher in outpatients than in controls but the IF was reversed. As in the previous studies, the slope of the correlation between the resting $\mathrm{LF} / \mathrm{HF}$ and $\mathrm{c}-\mathrm{MDT} / \mathrm{r}$ of the infra-umbilical $3 \mathrm{cpm}$ was positive in the controls (Homma, 2006), however, negative in the outpatients.

\section{Subjects and Methods}

This study enrolled the same medical students who volunteered to be the controls in the stress study involving the MDT (Homma, 2005; Homma, 2006; $\mathrm{n}=43$ ) together with additional volunteers $(\mathrm{n}=15)$. Thus there were 58 control subjects, 52 males and 6 females, ranging in age from 20-38. Outpatients ranged in age from 18-78 (39 \pm 16.8 , mean $\pm \mathrm{SE}, \mathrm{n}=25)$, with 16 females and 9 males. The outpatients had varied and unclassified diagnoses and their age and drug therapies were not taken into consideration in this study. With regard to the EGG parameters, this study focused on the dominant $3 \mathrm{cpm}$ activity. In addition, age matching between the control subjects and the outpatients was not possible because of limited outpatients and the limited duration of sampling. However, we have summarized the data as a basis for future study.

The methods of recording and analyzing EGGs were the same as in the previous studies (Homma et al., 1999; Homma, 2005). Unipolar EGGs were recorded from the 16 locations (channels, ch) from the thoraco-abdominal skin surface (see Fig. 1 of Homma, 2005, and also Homma et al., 2008), using a reference electrode on the right leg, with the approval of the ethics committee of Niigata University, Faculty of Medicine. Informed consent was obtained from each subject.

The amplifier was a modified electroencephalographic (EEG) amplifier, with a time constant of $5 \mathrm{~s}$, high cut $0.5 \mathrm{~Hz}$, low cut $-6 \mathrm{~dB}$ /oct and high cut $-12 \mathrm{~dB} /$ oct (Biotop 6R 12-4, NEC-Sanei, Japan). After cleaning the skin with ethanol, electrode cream was applied to the disc electrodes (11 $\mathrm{mm}$ diameter) for measurement of the EEG. The electrodes were fixed on the skin with surgical tape. Resting EGGs were recorded for about $20 \mathrm{~min}$, after the subjects had fasted for more than 8 hours and sampled for $128 \mathrm{~s}$ ( 1 file) every $1 \mathrm{~s}$ interval. After recording the resting controls, subjects were exposed to the stress of a MDT. The MDT involves tracing the cue figure of a metal star, which was reflected onto a mirror, by using an electric pen which gives a click alarm when the tracing runs off the edge of the star (an error). The MDT stress was undertaken for about 5 min to get 2-3 files of EGGs. After finishing the MDT test, a further 20 min of EGG recordings (about 7 files) were performed to obtain a running spectrum. The piled running spectra were obtained after the files were analyzed using the maximal entropy method (MEM). The spectral frequency readings were classified into five groups: the 1-cpm group (0$2.4 \mathrm{cpm})$, the 3-cpm group $(2.5-4.9 \mathrm{cpm})$, the 6-cpm group $(5.0-7.4 \mathrm{cpm})$, the 8-cpm group (7.5$9.9 \mathrm{cpm})$ and the 10-cpm group $(10.0-12.9 \mathrm{cpm})$. Ensemble means were obtained during rest, 
and both during and after the stress of performing the MDT. Spectral mean power amplitudes were calculated for each of the epigastric, supraumbilical and infraumbilical EGGs. With regard to the EGG parameters, this study focused mainly on the dominant spectral $3 \mathrm{cpm}$ frequency.

Electrocardiograms (ECGs) were recorded simultaneously with the EGGs for calculating the HRV. The LF $(0.039-0.148 \mathrm{~Hz}), \mathrm{HF}(0.148-0.398 \mathrm{~Hz})$ and LF/HF ratios were calculated from the R-R intervals (RRI) obtained by a special MEM analysis (Ambulatory ECG recorder, SM50, Fukuda Denshi, Japan). The respiratory frequency was monitored with a nasal thermistor. Representative running spectra with continuous LF/HF ratios are shown in Fig. 2 of a previous paper (Homma, 2005). The power content was defined as the power content of a certain frequency group to the total power of a spectrum. The power content ratio was defined as (power content during MDT / content during rest, c-MDT/r) of a certain frequency group. The anxiety and depression scores were estimated using HADS (hospital anxiety and depression scales) (Zigmond and Snaith, 1983; Mykletun et al., 2001). The mean and standard errors (SE) were calculated and the Student's $t$-test was used to determine statistical significance. $P$ values below 0.05 were considered to be significant.

\section{Results}

\section{Comparison of the basic parameters of ECG and respiratory rate}

No significant correlations were found between the age of outpatients and their anxiety scores, depression scores, heart rate, respiratory rate, and autonomic indices of LE, HF, and $\mathrm{LF} / \mathrm{HF}$ ratios in this study $(\mathrm{n}=25)$. The anxiety and depression scores, resting heart and respiratory rate, resting $\mathrm{LF}$, resting $\mathrm{HF}$, resting $\mathrm{LF} / \mathrm{HF}$ ratios, and those during the stress of performing the MDT for the outpatients and control subjects are listed in Table 1. As expected, the anxiety and depression scores were significantly higher in outpatients than in control subjects $(P<0.001-0.01)$. Similarly, the resting heart rate (Rest-BPM) and respiratory rate (RestResp.F.) were also significantly higher in outpatients $(P<0.001)$. The heart rate during the MDT stress (MDT-BPM) was also higher in outpatients $(P<0.05)$, but respiratory rate during MDT stress was higher in control subjects than in outpatients $(P<0.05)$. Resting LF/HF (Rest-LF/HF) was not significantly different between outpatients and control subjects, however, resting LF (Rest-LF) and HF (Rest-HF) were significantly lower in outpatients than in controls $(P<0.001)$.

Comparison of the spectral frequency, instability factor (IF) and power content ratio of the EGGs $(c-M D T / r)$

The spectral frequencies of the resting epigastric $3 \mathrm{cpm}(\mathrm{rCH} 1(3) \mathrm{Fr})$ were higher in outpatients than in controls $(P<0.05)$. In contrast, the IF of the resting supraumbilical $3 \mathrm{cpm}$ (rCH2(3)-IF) was higher in controls than in outpatients $(P<0.05$, Table 1$)$. No significant differences in the power content ratio of the MDT/r were found in the $3 \mathrm{cpm}$ groups.

\section{Comparison of the slopes of linear correlations of the EGG, ECG and HRV parameters}

The slopes and correlation coefficients of the EGG, ECG and HRV parameters are listed in Table 2. The slope of the linear correlation between anxiety and depression scores was positive 
Table 1. Comparison of the basic parameters of the ECG's, i.e., resting heart rate (rest-BPM), respiratory rate (rest-Resp.F.), resting LF (rest-LF), resting HF (rest-HF), resting LF/HF (rest-LF/HF) and of the EGG's, i.e. epigastric resting frequency ( $\mathrm{rCH} 1(3) \mathrm{Fr})$ and resting supraumbilical instability factor of the $3 \mathrm{cpm}$ frequency (rCH2(3)-IF)

\begin{tabular}{lccc}
\hline & Outpatients & Controls & $P$ values \\
\hline Age & $18-78$ & $19-38$ & \\
Anxiety & $39 \pm 16.8(\mathrm{n}=25)$ & $(\mathrm{n}=58)$ & \\
Depression & $8.0 \pm 0.88$ & $3.8 \pm 0.32$ & $P<0.01$ \\
Rest-BPM & $7.2 \pm 0.73$ & $3.2 \pm 0.29$ & $P<0.001$ \\
Rest-Resp.F. & $69.3 \pm 2.62$ & $58.6 \pm 0.98$ & $P<0.01$ \\
Rest-LF & $16.4 \pm 0.58$ & $12.8 \pm 0.35$ & $P<0.001$ \\
Rest-HF & $499 \pm 87$ & $1694 \pm 170$ & $P<0.001$ \\
Rest-LF/HF & $526 \pm 107$ & $1395 \pm 136$ & $P<0.001$ \\
rCH1(3)Fr & $1.95 \pm 0.31$ & $1.66 \pm 0.16$ & $\mathrm{NS}$ \\
rCH2(3)-IF & $3.28 \pm 0.08$ & $3.03 \pm 0.04$ & $P<0.05$ \\
\end{tabular}

Table 2. Heart rate and EGG responses during the stress of the mirror drawing test (MDT). Linear relationships between both the heart rate (BPM), respiratory frequency (Resp.F.), and EGGindices. Anxiety (A), resting LF/HF EGG indices include the peak power ratio (p-MDT/f) and area power content ratio (area MDT/f). $P$, statistical probability. $\alpha$, slope; $\mathrm{R}_{2}$, correlation coefficient; $\mathrm{CH} 1$, epigastric EGG.; $\mathrm{CH} 2$, supraumbilical EGG.; CH3, infraumbilical EGG. n=2225 for outpatients, $57-58$ for controls

\begin{tabular}{lcccccccc}
\hline & \multicolumn{3}{c}{ Outpatients } & & \multicolumn{3}{c}{ Controls } \\
\cline { 2 - 3 } & $P$ & $\alpha$ & $\mathrm{R}_{2}$ & & $P$ & $\alpha$ & $\mathrm{R}_{2}$ \\
\hline A-D & $<0.01$ & 0.46 & 0.31 & & $<0.001$ & 0.45 & 0.29 \\
Rest-HF - rest-BPM & $<0.001$ & -0.045 & & 0.40 & & $<0.01$ & -0.02 & 0.12 \\
Rest-HF - MDT-BPM & $<0.001$ & -0.015 & & 0.49 & & $<0.01$ & -0.003 & 0.12 \\
Power content-MDT/r (c-MDT/r) & & & & & & & \\
Rest-LF/HF - CH3(3) & $<0.05$ & -1.15 & & 0.165 & & $<0.05$ & 0.126 & 0.072
\end{tabular}

in both outpatients and controls. The slope of the significant linear correlation between resting HF (Rest-HF) and resting heart rate (Rest-BPM) and between resting HF (Rest-HF) and heart rate during MDT (MDT-BPM) was negative. In contrast, the slope of significant linear correlation was negative in outpatients but was positive in controls when comparing the resting $\mathrm{LF} / \mathrm{HF}$ ratios (Rest-LF/HF) and infraumbilical power content ratios of the $3 \mathrm{cpm}$ during the MDT to that at rest (CH3(3) c-MDT/r).

\section{Discussion}

It is generally known that baroreflex sensitivity, LF, and HF (Barantke et al., 2008; Laitinen et al., 2004) and LF/HF (Laitinen et al., 2004) continuously decline with age. Therefore, the ages of subjects in both control and outpatient groups and age-matching need to be taken into account. However, no significant correlations were found between the age of outpatients and their anxiety scores, depression scores, heart rate, respiratory rate, and autonomic indices of 
$\mathrm{LF}, \mathrm{HF}$, and LF/HF in this study $(\mathrm{n}=25)$. Therefore, differences of autonomic indices between the subjects may not be dependent on age.

The anxiety and depression scores of outpatients were significantly higher than those of controls as expected $(P<0.001-0.01)$. The linear correlation between the anxiety and depression scores had a similar slope and correlation coefficient both in the controls and outpatients in this study. Individuals with higher levels of phobic anxiety have a higher resting heart rate (Kawachi et al., 1995). Similarly, the heart rate is significantly higher in those with higher traitanxiety scores and high trait anxiety is associated with significantly reduced vagal control of heart rate, in comparison to low trait anxiety, indicated by a reduction in the baroreflex control of the heart rate and respiratory sinus arrhythmia (Watkins et al., 1998). Similarly, those outpatients with higher anxiety scores had a higher resting heart rate and respiratory rate than controls and the anxiety scores and resting heart rate while performing the MDT had a positive slope demonstrating an insignificant linear correlation both in controls and outpatients in this study (not shown in Table 2).

$\mathrm{LF}$ and $\mathrm{HF}$ are both considered to reflect the sympathetic and parasympathetic tone, respectively, and the ratio of LF/HF reflects the sympatho-vagal balance (Pagani et al., 1986; Pagani et al., 1991; American Heart Association, 1996). Groups with higher anxiety symptoms have significantly lower resting values for all power spectral components of heart rate variability, $\mathrm{LF}, \mathrm{HF}$ and the $\mathrm{LF} / \mathrm{HF}$ ratio and the resting $\mathrm{LF} / \mathrm{HF}$ ratio is positively correlated with the anxiety symptom scores. In addition, the LF/HF ratio is positively correlated with anxiety symptom scores at rest and the anxiety disorders are marked by either a low basal heart rate variability or a reduced vagal and an increased sympathetic cardiac responsiveness (Yeragani et al., 1990; Friedman and Thayer, 1998). However, during tilt stress, either the LF/HF ratio correlated negatively with the anxiety scores or a high basal sympathetic activity blunted sympathetic responses in high anxious subjects (Piccirillo et al., 1997; Friedman, 2007). Similarly, the current study found significantly lower LF and HF values and insignificantly higher LF/HF ratios in outpatients than in controls (Table 1). No significant positive or negative linear relationship was observed between the anxiety scores and LF, HF in the controls and outpatients. However there was a negative slope in the outpatients but a positive slope in the control between the resting $\mathrm{LF} / \mathrm{HF}$ ratios and the power content ratios of the $3 \mathrm{cpm}$ frequencies in their infraumbilical EGG during the MDT to rest period (c-MDT/r) (Table 2). Therefore, higher anxiety and LF/HF ratios might produce reduced stress responses in the EGG, probably due to colonic electrical activity, because $3 \mathrm{cpm}$ frequency activity in the infraumbilical EGG might reflect colonic electrical activities (Pezzola et al., 1989; Sarna, 1989; Homma et al., 1995; Homma et al., 1999; Amaris et al., 2002). Arithmetic task stress induces a significant increase in dominant gastric power and a slight decrease in dominant gastric frequency in healthy subjects (Riezzo et al., 1996). Positive slopes were observed between the resting LF/HF ratios and the $\mathrm{cMDT} / \mathrm{r}$ ratios in controls. In addition, a significantly higher spectral frequency during MDT stress was found in the $3 \mathrm{cpm}$ frequency activity in the infraumbilical EGG in outpatients $(P<0.05)$ and a significantly higher IF of the resting $3 \mathrm{cpm}$ frequency activity in the infraumbilical EGG in controls (Table 1). It is interesting to note that relatively lower anxiety increased the heart rate and in contrast, relatively higher anxiety decreased the heart rate. 
Similarly, those outpatients with significantly higher anxiety responded with a negative slope to the MDT stress, and those control subjects with significantly lower anxiety responded with a positive slope to the MDT. Once again, the averaged resting LF/HF ratios were similar and not significantly different between controls and outpatients but the resting LF and HF were significantly lower in outpatients (Table 1). It is reported that the subjects with higher anxiety during the presentation of signals predicting noxious thermal stimuli showed lower heart rate changes than those with lower anxiety (Ploghaus et al., 2001). The response capacity of the limbic and related autonomic systems are facilitated in lower anxiety and occluded or inhibited in higher anxiety in response to the MDT stress. This is reminiscent of the classic concepts of the subliminal fringe (Sherrington, 1929), post tetanic potentiation (PTP) or presynaptic inhibition (Eccles et al., 1962; Bliss and Lomo, 1973; Bliss and Gardner-Medwin, 1973; Dudek and Bear, 1992; Mulkey and Malenka, 1992).

The facilitation or depression of MDT stress on the gastric and colonic myoelectric activity of the EGG's and the linear correlations between the resting LF/HF ratios and stress responses were reported in previous papers (Homma, 2005; Homma, 2006). In this study, a significant linear correlation was found between rest-LF/HF ratios and the colonic MDT stress responses (c-MDT/r) but not in the stomach stress responses (Table 2). These results may be explained by suggesting that the various stressors depress stomach contractility and emptying and facilitate colonic motility, transit and defecation via corticotrophin-releasing factor receptors 2 (CRF-R2) and CRF-R1, respectively (Tache et al., 2001; Bhatia and Tandon, 2005).

In summary, the anxiety scores, depression scores, resting heart rate and resting respiratory rate were significantly higher in outpatients than in controls. Interestingly, the slope of the linear correlation between the resting-LF/HF ratios and the power content ratios of c$\mathrm{MDT} / \mathrm{r}$ of the $3 \mathrm{cpm}$ requencies of the infraumbilical EGG's is positive in controls with a relatively lower anxiety but negative in outpatients with a relatively higher anxiety level. The colonic response capacity to stress may therefore decrease in individuals with a higher anxiety level.

\section{References}

Amaris, M.A., Sanmiguel, C.P., Sadowski, D.C., Bowes, K. and Mintchev, M.P. (2002). Electrical activity from colon overlaps with normal gastric electrical activity in cutaneous recordings. Dig. Dis. Sci. 47: 2480-2485.

Barantke, M., Krauss, T., Ortak, J., Lieb, W., Reppel, W., Burgdorf, C., Pramstaller, P.P., Schunkert, H. and Bonnemeier, M. (2008). Effects of gender and aging on differential autonomic responses to orthostatic maneuvers. J. Cardiovasc. Electrophysiol. 19: 1296-1303.

Bhatia, V. and Tandon, R.K. (2005). Stress and the gastrointestinal tract. J. Gastroenterol. Hepatol. 20: 332-333.

Bliss, T.V. and Lomo, T. (1973). Long-lasting potentiation of synaptic transmission in the perforant path. J. Physiol. (Lond.) 232: 331-356.

Bliss, T.V. and Gardner-Medwin, A.R. (1973). Long-lasting potentiation of synaptic transmission in the dentate area of the unanesthetized rabbit following stimulation of the perforant path. J. Physiol. (Lond.) 232: 357-374.

Dudek, S.M. and Bear, M.F. (1992). Homosynaptic long-term depression in area CA1 of hippocampus 
and effects of N-methyl-D-aspartate receptor blockade. Proc. Natl. Acad. Sci. USA. 89: 43634367.

Eccles, J.C., Kostyuk, P.G. and Schmidt, R.F. (1962). Presynaptic inhibition of the central actions of flexor reflex afferents. J. Physiol. (Lond.) 161: 258-281.

Friedman, B.H. and Thayer, J.F. (1998). Autonomic balance revisited: panic anxiety and heart rate variability. J. Psychosomatic Res. 44: 133-151.

Friedman, B.H. (2007). An autonomic flexibility-neurovisceral integration model of anxiety and cardiac vagal tone. Biol. Psychol. 74: 185-199.

Homma, S. (2005). The effects of stress in response to mirror drawing test trials on the electrogastrogram, heart rate and respiratory rate of human subjects. J. Smooth Muscle Res. 41: 221-233.

Homma, S. (2006). Correlations between the responses of electrogastrograms, heart rate and respiratory rate to the stress of the mirror drawing test in human subjects. J. Smooth Muscle Res. 42: 9-19.

Homma, S., Hasegawa, J., Maruta, T., Watanabe N., Matsuo, H., Tamiya, Y., Nishimaki, T., Suzuki, T., Muto, T. and Hatakeyama, K. (1999). Isopower maps of the electrogastrogram (EGG) after total gastrectomy or total colectomy. Neurogastroenterol. Motil. 11: 441-448.

Homma, S., Kobayashi, Y., Kosugi, S., Ohashi, M., Kanda, T., Okamoto, H. and Hatakeyama, K. (2008). Postoperative reorganization of gastric pacemaker activity in patients after an extended period following distal gastrectomy. J. Smooth Muscle Res. 44: 113-122.

Homma, S., Shimakage, N., Yagi, M., Hasegawa, J., Sato, K., Matsuo, H., Tamiya, Y., Tanaka, O., Muto, T. and Hatakeyama, K. (1995). Electrogastrography prior to and following total gastrectomy, subtotal gastrectomy, and gastric tube formation. Dig. Dis. Sci. 40: 893-900.

Kawachi, I., Sparrow, D., Vokonas, P.S. and Weiss, S.T. (1995). Decreased heart rate variability in men with phobic anxiety (data from the normative aging study). Am. J. Cardiol. 75: 882-885.

Laitinen, T., Niskanen, L., Geelen, G., Lansimies, E. and Hartikainen, J. (2004). Age dependency of cardiovascular autonomic responses to head-up tilt in healthy subjects. J. Appl. Physiol. 96: 2333-2340.

Mulkey, R.M. and Malenka, R.C. (1992). Mechanisms underlying of homosynaptic long-term depression in area CA1 of the hippocampus. Neuron 9: 965-975.

Mykletun, A., Stordal, E. and Dahl, A.A. (2001). Hospital anxiety and depression (HAD) scale: factor structure, item analyses and internal consistency in a large population. Br. J. Psychiatr. 179: 540-544.

Pagani, M., Lombardi, F., Guzzetti, S., Rimoldi, O., Furlan, R., Pizzinelli, P., Sandrone, G., Malfatto, G., Dell'Orto, S., Piccaluga, E., Turiel, M., Baselli, G., Cerutti, S. and Maliani, A. (1986). Power spectral analysis of heart rate and arterial pressure variabilities as a marker of sympathovagal interaction in man and conscious dog. Circ. Res. 59: 178-193.

Pagani, M., Mazzuero, G., Ferrari, A., Liberati, D., Ceruti, S., Vaitl, D., Tavazzi, L. and Malliani, A. (1991). Sympathovagal interaction during mental stress. Circul. 83: 434-451.

Pezzola, F., Riezzo, G., Maselli, M.A. and Giorgio, I. (1989). Electrical activity recorded from abdimal surface after gastrectomy or colectomy. Gastroenterol. 97: 313-320.

Piccirillo, G., Elvira, S., Bucca, C., Viola, E., Cacciafesta, M. and Marogliano, V. (1997). Abnormal passive head-up tilt test in subjects with symptoms of anxiety power spectral analysis study of heart rate and blood pressure. Int. J. Cardiol. 60: 121-131.

Ploghaus, A., Narain, C., Beckmann, C.F., Clare, S., Bantick, S., Wise, R., Matthews, P.M., Nicholas, J., Rawlims, P. and Tracey, I. (2001). Exacerbation of pain by anxiety is associated with activity in a hippocampal network. J. Neurosci. 21: 9896-9903.

Riezzo, G., Porcelli, P., Guerra, V. and Giorgio, I. (1996). Effects of different psychophysiological stressors on the cutaneous electrogastrogram in healthy subjects. Arch. Physiol. Biochem. 104: 282-286. 
Sarna, S.K. (1989). Colonic electrical activity as an indicator of colonic motor function. In: Gastrointestinal Motility: What test?, ed. by N.W. Read, Wrightston Biomedical Publishers, Petersfield, UK, pp. 203-211.

Sherrington, C. (1929). Some functional probrems attaching to convergence. Proc. Roy. Soc. Lond. 105B: 332-362.

Tache, Y., Martinez, V., Million, M. and Wang, L. (2001). Stress and the gastrointestinal tract III. Stressrelated alterations of gut motor functions: role of brain corticotropin-releasing factor receptors. Am. J. Physiol. 280: G173-G177.

Watkins, L.L., Grossman, P., Krishnan, R. and Sherwood, A. (1998). Anxiety and vagal control of heart rate. Psychosom. Med. 60: 498-502.

Yeragani, V.K., Balon, R., Pohl, R., Ramesh, C., Glitz, D., Weinberg, P. and Merlos, B. (1990). Decreased R-R variance in panic disorder patients. Acta Psychiatr. Scand. 81: 554-559.

Zigmond, A.S. and Snaith, R.P. (1983). The hospital anxiety and depressionscale. Acta Psychiatr. Scand. 67: 361-370.

Task force of the European Society of Cardiology and the North American Society of Pacing and Electrophysiology (1966). HRV. Standards of measurement, physiological interpretation, and clinical use. Circlation 93: 1043-1065. 